\title{
Certain Subclasses of Bi-Univalent and Meromorphic Functions Defined By Al-Oboudi Differential Operator
}

\author{
D. D. Bobalade ${ }^{1}$ and N. D. Sangle ${ }^{2}$ \\ ${ }^{1}$ Department of Mathematics, Shivaji University, Kolhapur, Maharashtra, India- 416004 \\ ${ }^{2}$ Department of Mathematics, D.Y. Patil College of Engineering and Technology \\ Kasaba Bawada, Kolhapur, Maharashtra, India 416006 \\ Email: 1dnyaneshwar.boblade@gmail.com, 2navneet_sangle@rediffmail.com
}

\begin{abstract}
In this paper, we introduce a subclass $\sum_{M}^{h, p}(k, \delta, \mu, \lambda)$ of bi-univalent and meromorphic functions by using Al-Oboudi differential operator on $\Delta=\{z \in \mathbb{C}: 1<|z|<\infty\}$. Also we obtain bounds of coefficients $\left|b_{0}\right|$ and $\left|b_{1}\right|$ for functions belongs to $\sum_{M}^{h, p}(k, \delta, \mu, \lambda)$. The results obtained in this paper are more better and generalized of previous results of various author.
\end{abstract}

Keywords: Harmonic functions, Univalent functions, Differential operator, Extreme points.

2010 Mathematics Subject Classification: 30C45, 30C50.

\section{Introduction}

Let $\Sigma$ be the class of functions $f$ of the form

$$
f(z)=z+\sum_{l=0}^{\infty} \frac{b_{l}}{z^{l}},
$$

which are meromorphic univalent in the domain $\Delta=\{z \in \mathbb{C}: 1<|z|<\infty\}$. Since every function $f$ belong to $\Sigma$ has an inverse function $f^{-1}$ exist and inverse function satisfies conditions:

and

$$
f^{-1}(f(z))=z(z \in \Delta)
$$

$$
f\left(f^{-1}(w)\right)=w, w \in \Delta \quad(M<|w|<\infty, M>0),
$$

where

$$
f^{-1}(w)=q(w)=w-b_{0}-\frac{b_{1}}{w}-\frac{b_{2}+b_{0} b_{1}}{w^{2}}-\frac{b_{3}+2 b_{0} b_{2}+b_{0}^{2} b_{1}+b_{1}^{2}}{w^{3}}+\cdots .
$$

A function $f \in \Sigma$ is said to be meromorphic bi-univalent in $\Delta$ if both $f$ and $f^{-1}$ are meromorphic univalent in $\Delta$. The class of meromorphic bi-univalent functions of the form (1) in $\Delta$ is denoted by $\Sigma_{M}$.

Srivastava et al. [17], Safa Salehian and Ahmad Zireh [12], Hamidi et al. [7], Amol Patil and Uday Naik [11] and many other researchers (see $[4,5,8,9,10,14,15,18]$ ) have introduced new subclasses of meromorphically bi-univalent functions and obtained estimates on the initial coefficients for functions in each of these subclasses.

Let $\mathcal{A}$ denote the class of analytic functions $h(z)$ of the form

$$
h(z)=z+\sum_{l=2}^{\infty} a_{l} z^{l}
$$

defined in the unit disc $\mathbb{U}=\{z \in \mathbb{C}:|z|<1\}$ with normalization $h(0)=h_{z}(0)-1=0$. Let the class of all normalized analytic univalent functions in the unit disc $\mathbb{U}$ is denoted by $S$. A function $h \in \mathcal{A}$ is said to 
be bi-univalent in $\mathbb{U}$ if both $h$ and $h^{-1}$ are univalent in $\mathbb{U}$. Let the class of analytic bi-univalent functions is denoted by $\Sigma^{\prime}$. Brannan and Taha [2], Srivastava et al. [16] and many other researchers (see [3, 6]) introduced certain subclasses of bi-univalent function class $\Sigma^{\prime}$.

Now, Al-Oboudi [1] introduced the Al-Oboudi operator $D_{\delta}^{k}: \mathcal{A} \rightarrow \mathcal{A}$ and defined as

$$
D^{k} h(z)=D_{\delta}^{k} h(z)=z+\sum_{l=2}^{\infty}[1+(l-1) \delta]^{k} a_{l} z^{l}, \quad k \in \mathbb{N}_{0}=\mathbb{N} \cup\{0\}, \delta \geq 0,
$$

where $h \in \mathcal{A}$ of the form (3).

Amol Patil and Uday Naik [11] extend the Al-Oboudi operator $D_{\delta}^{k}: \Sigma \rightarrow \Sigma$ and defined as $D^{k} f(z)=D_{\delta}^{k} f(z)=z+(1-\delta)^{k} b_{0}+\sum_{l=1}^{\infty}[1-(l+1) \delta]^{k} b_{l} z^{-l}, \quad k \in \mathbb{N}_{0}=\mathbb{N} \cup\{0\}, \delta>1$, where $f \in \Sigma$ of the form (1).

In 2019, Saideh Hajiparvaneh and Ahmad Zireh [13] define the subclass $\Sigma_{M}^{h, p}(\mu, \lambda)$ consisting of meromorphic functions $f(z)$ of the form (1) satisfies the following conditions:

$$
f \in \Sigma_{M},\left[(1-\lambda)\left(\frac{f(z)}{z}\right)^{\mu}+\lambda f^{\prime}(z)\left(\frac{f(z)}{z}\right)^{\mu-1}\right] \in h(\Delta)
$$

and

$$
\left[(1-\lambda)\left(\frac{q(w)}{w}\right)^{\mu}+\lambda q^{\prime}(w)\left(\frac{q(w)}{w}\right)^{\mu-1}\right] \in p(\Delta),
$$

where $q$ is function given by (2).

Motivated by the aforecited works, we introduce new subclasses of bi-univalent and meromorphic functions by using Al-Oboudi Differential operator. Also obtain the coefficient bounds $\left|b_{0}\right|$ and $\left|b_{1}\right|$ for functions in this new subclasses.

\section{Coefficient Estimates}

Definition 2.1 Let the analytic functions $\mathrm{h}, \mathrm{p}: \Delta \rightarrow \mathbb{C}$ be

such that

$$
\mathrm{h}(\mathrm{z})=1+\frac{\mathrm{h}_{1}}{\mathrm{z}}+\frac{\mathrm{h}_{2}}{\mathrm{z}^{2}}+\frac{\mathrm{h}_{3}}{\mathrm{z}^{3}}+\cdots, \quad \mathrm{p}(\mathrm{z})=1+\frac{\mathrm{p}_{1}}{\mathrm{z}}+\frac{\mathrm{p}_{2}}{\mathrm{z}^{2}}+\frac{\mathrm{p}_{3}}{\mathrm{z}^{3}}+\cdots,
$$

$$
\min \{\Re(\mathrm{h}(\mathrm{z})), \Re(\mathrm{p}(\mathrm{z}))\}>0 \quad(z \in \Delta) .
$$

Definition 2.2 A function $\mathrm{f}(\mathrm{z})$ of the form (1) is said to be in the class $\Sigma_{\mathrm{M}}^{\mathrm{h}, \mathrm{p}}(\mathrm{k}, \delta, \mu, \lambda), \mathrm{k} \in \mathbb{N}_{0}=\mathbb{N} U$ $\{0\}, \lambda \geq 1, \lambda>\mu, \mu \geq 0$ and $\delta>1$ if satisfies the following conditions :

$$
f \in \Sigma_{M},\left[(1-\lambda)\left(\frac{D_{\delta}^{k} f(z)}{z}\right)^{\mu}+\lambda\left(D_{\delta}^{k} f(z)\right)^{\prime}\left(\frac{D_{\delta}^{k} f(z)}{z}\right)^{\mu-1}\right] \in h(\Delta)
$$

and

$$
\left[(1-\lambda)\left(\frac{D_{\delta}^{k} q(w)}{w}\right)^{\mu}+\lambda\left(D_{\delta}^{k} q(w)\right)^{\prime}\left(\frac{D_{\delta}^{k} q(w)}{w}\right)^{\mu-1}\right] \in p(\Delta),
$$

where $q$ is the function given by (2).

For $k=0$, the class $\Sigma_{M}^{h, p}(k, \delta, \mu, \lambda)$ become $\Sigma_{M}^{h, p}(\mu, \lambda)$, studied by Saideh Hajiparvaneh and Ahmad Zireh [13].

Remark 2.1 For various choices of $h$ and $p$, we get various subclasses of class $\Sigma_{M}^{h, p}(k, \delta, \mu, \lambda)$ as follows:

$$
\text { If take } h(z)=p(z)=\left(\frac{1+\frac{1}{z}}{1-\frac{1}{z}}\right)^{\alpha}=1+\frac{2 \alpha}{z}+\frac{2 \alpha^{2}}{z^{2}}+\cdots \quad(0<\alpha \leq 1, z \in \Delta) \text { in Definition 2.2, then }
$$

we get subclass $\Sigma_{M}^{h, p}(k, \delta, \mu, \lambda)=\Sigma_{M}^{*}(k, \delta, \mu, \lambda, \alpha)$, studied by Bobalade and Sangle [4].

Definition 2.3 [4] A function $\mathrm{f}(\mathrm{z}) \in \Sigma_{\mathrm{M}}^{\mathrm{h}, \mathrm{p}}(\mathrm{k}, \delta, \mu, \lambda)$ of the form (1) belongs to the class $\Sigma_{\mathrm{M}}^{*}(\mathrm{k}, \delta, \mu, \lambda, \alpha)$ if

$$
\mathrm{f} \in \Sigma_{\mathrm{M}},\left|\arg \left[(1-\lambda)\left(\frac{\mathrm{D}_{\delta}^{\mathrm{k}} \mathrm{f}(\mathrm{z})}{\mathrm{z}}\right)^{\mu}+\lambda\left(\mathrm{D}_{\delta}^{\mathrm{k}} \mathrm{f}(\mathrm{z})\right)^{\prime}\left(\frac{\mathrm{D}_{\delta}^{\mathrm{k}} \mathrm{f}(\mathrm{z})}{\mathrm{z}}\right)^{\mu-1}\right]\right|<\frac{\alpha \pi}{2} \quad(\mathrm{z} \in \Delta)
$$


and

$$
\left|\arg \left[(1-\lambda)\left(\frac{\mathrm{D}_{\delta}^{\mathrm{k}} \mathrm{q}(\mathrm{w})}{\mathrm{w}}\right)^{\mu}+\lambda\left(\mathrm{D}_{\delta}^{\mathrm{k}} \mathrm{q}(\mathrm{w})\right)^{\prime}\left(\frac{\mathrm{D}_{\delta}^{\mathrm{k}} \mathrm{q}(\mathrm{w})}{\mathrm{w}}\right)^{\mu-1}\right]\right|<\frac{\alpha \pi}{2} \quad(\mathrm{w} \in \Delta),
$$

where $\mathrm{k} \in \mathbb{N}_{0}=\mathbb{N} \cup\{0\}, \lambda \geq 1, \lambda>\mu, \mu \geq 0, \delta>1$ and $0<\alpha \leq 1$.

Remark 2.2 If take $h(z)=p(z)=\frac{1+\frac{(1-2 \beta)}{z}}{1-\frac{1}{z}}=1+\frac{2(1-\beta)}{z}+\frac{2(1-\beta)}{z^{2}}+\cdots \quad(0 \leq \beta<1, z \in \Delta)$ in Definition 2.2, then we get subclass $\Sigma_{M}^{h, p}(k, \delta, \mu, \lambda)=\Sigma_{M}^{*}(k, \delta, \mu, \lambda, \beta)$, studied by Bobalade and Sangle [4].

Definition 2.4 [4] A function $\mathrm{f}(\mathrm{z}) \in \Sigma_{\mathrm{M}}^{\mathrm{h}, \mathrm{p}}(\mathrm{k}, \delta, \mu, \lambda)$ of the form (1) belongs to the class $\Sigma_{\mathrm{M}}^{*}(\mathrm{k}, \delta, \mu, \lambda, \beta)$ if

$$
\mathrm{f} \in \Sigma_{\mathrm{M}}, \Re\left[(1-\lambda)\left(\frac{\mathrm{D}_{\delta}^{\mathrm{k}} \mathrm{f}(\mathrm{z})}{\mathrm{z}}\right)^{\mu}+\lambda\left(\mathrm{D}_{\delta}^{\mathrm{k}} \mathrm{f}(\mathrm{z})\right)^{\prime}\left(\frac{\mathrm{D}_{\delta}^{\mathrm{k}} \mathrm{f}(\mathrm{z})}{\mathrm{z}}\right)^{\mu-1}\right]>\beta \quad(\mathrm{z} \in \Delta)
$$

and

$$
\Re\left[(1-\lambda)\left(\frac{D_{\delta}^{\mathrm{k}} \mathrm{q}(\mathrm{w})}{\mathrm{w}}\right)^{\mu}+\lambda\left(\mathrm{D}_{\delta}^{\mathrm{k}} \mathrm{q}(\mathrm{w})\right)^{\prime}\left(\frac{\mathrm{D}_{\delta}^{\mathrm{k}} \mathrm{q}(\mathrm{w})}{\mathrm{w}}\right)^{\mu-1}\right]>\beta \quad(\mathrm{w} \in \Delta),
$$

where $\mathrm{k} \in \mathbb{N}_{0}=\mathbb{N} \cup\{0\}, \lambda \geq 1, \lambda>\mu, \mu \geq 0, \delta>1$ and $0 \leq \beta<1$.

If we put $k=0$ in the classes $\Sigma_{M}^{*}(k, \delta, \mu, \lambda, \alpha)$ and $\Sigma_{M}^{*}(k, \delta, \mu, \lambda, \beta)$, then we get two classes $\Sigma_{M}^{*}(\mu, \lambda, \alpha)$ and $\Sigma_{M}^{*}(\mu, \lambda, \beta)$ respectively, studied by Orhan et al. [10].

Theorem 2.1 Let $f(z)$ of the form (1) belong to the class $\Sigma_{M}^{h, p}(k, \delta, \mu, \lambda)$. Then

and

$$
\left|b_{0}\right| \leq \min \left[\sqrt{\frac{\left|h_{1}\right|^{2}+\left|p_{1}\right|^{2}}{2(\mu-\lambda)^{2}(1-\delta)^{2 k}}}, \sqrt{\frac{\left|h_{2}\right|+\left|p_{2}\right|}{(\mu-2 \lambda)(\mu-1)(1-\delta)^{2 k} \mid}}\right]
$$

$$
\left|b_{1}\right| \leq \min \left[\frac{\left|h_{2}\right|+\left|p_{2}\right|}{2\left|(\mu-2 \lambda)(1-2 \delta)^{k}\right|}, \sqrt{\frac{\left|h_{2}\right|^{2}+\left|p_{2}\right|^{2}}{2(\mu-2 \lambda)^{2}(1-2 \delta)^{2 k}}+\frac{(\mu-1)^{2}\left(\left|h_{1}\right|^{2}+\left|p_{1}\right|^{2}\right)^{2}}{16(\mu-\lambda)^{4}(1-2 \delta)^{2 k}}}\right] .
$$

Proof. From conditions (4) and (5), we have

and

$$
(1-\lambda)\left(\frac{D_{\delta}^{k} f(z)}{z}\right)^{\mu}+\lambda\left(D_{\delta}^{k} f(z)\right)^{\prime}\left(\frac{D_{\delta}^{k} f(z)}{z}\right)^{\mu-1}=h(z)(z \in \Delta)
$$

$$
(1-\lambda)\left(\frac{D_{\delta}^{k} q(w)}{w}\right)^{\mu}+\lambda\left(D_{\delta}^{k} q(w)\right)^{\prime}\left(\frac{D_{\delta}^{k} q(w)}{w}\right)^{\mu-1}=p(w)(w \in \Delta),
$$

where $h(z)$ and $p(w)$ are functions such that it's real part positive in $\Delta$ and have forms

$$
h(z)=1+\frac{h_{1}}{z}+\frac{h_{2}}{z^{2}}+\frac{h_{3}}{z^{3}}+\cdots
$$

and

Implies

$$
p(w)=1+\frac{p_{1}}{w}+\frac{p_{2}}{w^{2}}+\frac{p_{3}}{w^{3}}+\cdots
$$

$$
(1-\lambda)\left(\frac{D_{\delta}^{k} f(z)}{z}\right)^{\mu}+\lambda\left(D_{\delta}^{k} f(z)\right)^{\prime}\left(\frac{D_{\delta}^{k} f(z)}{z}\right)^{\mu-1}=1+\frac{h_{1}}{z}+\frac{h_{2}}{z^{2}}+\frac{h_{3}}{z^{3}}+\cdots,(z \in \Delta)
$$

and

$$
(1-\lambda)\left(\frac{D_{\delta}^{k} q(w)}{w}\right)^{\mu}+\lambda\left(D_{\delta}^{k} q(w)\right)^{\prime}\left(\frac{D_{\delta}^{k} q(w)}{w}\right)^{\mu-1}=1+\frac{p_{1}}{w}+\frac{p_{2}}{w^{2}}+\frac{p_{3}}{w^{3}}+\cdots(w \in \Delta) .
$$

Now, equating the coefficients in equation (12) and (13), we obtain

$$
\begin{gathered}
(\mu-\lambda)(1-\delta)^{k} b_{0}=h_{1}, \\
(\mu-2 \lambda)\left[(1-2 \delta)^{k} b_{1}+\left(\frac{\mu-1}{2}\right)(1-\delta)^{2 k} b_{0}^{2}\right]=h_{2},
\end{gathered}
$$




$$
-(\mu-\lambda)(1-\delta)^{k} b_{0}=p_{1}
$$

and

$$
(\mu-2 \lambda)\left[-(1-2 \delta)^{k} b_{1}+\left(\frac{\mu-1}{2}\right)(1-\delta)^{2 k} b_{0}^{2}\right]=p_{2} .
$$

From equation (14) and equation (17), we get

$$
h_{1}=-p_{1}
$$

and

$$
2(\mu-\lambda)^{2}(1-\delta)^{2 k} b_{0}^{2}=h_{1}^{2}+p_{1}^{2} .
$$

By adding equation (15) to equation (17), we get

$$
(\mu-2 \lambda)(\mu-1)(1-\delta)^{2 k} b_{0}^{2}=h_{2}+p_{2} .
$$

Therefore, From equation (19), we get

$$
b_{0}^{2}=\frac{h_{1}^{2}+p_{1}^{2}}{2(\mu-\lambda)^{2}(1-\delta)^{2 k}}
$$

and from equation (20), we get

$$
b_{0}^{2}=\frac{h_{2}+p_{2}}{(\mu-2 \lambda)(\mu-1)(1-\delta)^{2 k}} .
$$

Hence, from (21) and (22), we find that

$$
\left|b_{0}\right|^{2} \leq \frac{\left|h_{1}\right|^{2}+\left|p_{1}\right|^{2}}{2(\mu-\lambda)^{2}(1-\delta)^{2 k}}
$$

and

Hence

$$
\left|b_{0}\right|^{2} \leq \frac{\left|h_{2}\right|+\left|p_{2}\right|}{\left|(\mu-2 \lambda)(\mu-1)(1-\delta)^{2 k}\right|}
$$

$$
\left|b_{0}\right| \leq \min \left[\sqrt{\frac{\left|h_{1}\right|^{2}+\left|p_{1}\right|^{2}}{2(\mu-\lambda)^{2}(1-\delta)^{2 k}}}, \sqrt{\frac{\left|h_{2}\right|+\left|p_{2}\right|}{\left|(\mu-2 \lambda)(\mu-1)(1-\delta)^{2 k}\right|}}\right] .
$$

Now, subtracting equation (17) from equation (15), we obtain

$$
2(\mu-2 \lambda)(1-2 \delta)^{k} b_{1}=h_{2}-p_{2} .
$$

By squaring and adding equations (15) and (17), we get new equation. Using equation (19) in new equation, we obtain

$$
b_{1}^{2}=\frac{h_{2}^{2}+p_{2}^{2}}{2(\mu-2 \lambda)^{2}(1-2 \delta)^{2 k}}+\frac{(\mu-1)^{2}\left(h_{1}^{2}+p_{1}^{2}\right)^{2}}{16(\mu-\lambda)^{4}(1-2 \delta)^{2 k}} .
$$

By using equations (10), (11) in equation (23) and (24), finally we yield

and

$$
\left|b_{1}\right| \leq \frac{\left|h_{2}\right|+\left|p_{2}\right|}{2\left|(\mu-2 \lambda)(1-2 \delta)^{k}\right|}
$$

$$
\left|b_{1}\right| \leq \sqrt{\frac{\left|h_{2}\right|^{2}+\left|p_{2}\right|^{2}}{2(\mu-2 \lambda)^{2}(1-2 \delta)^{2 k}}+\frac{(\mu-1)^{2}\left(\left|h_{1}\right|^{2}+\left|p_{1}\right|^{2}\right)^{2}}{16(\mu-\lambda)^{4}(1-2 \delta)^{2 k}}} .
$$

Hence

$$
\left|b_{1}\right| \leq \min \left[\frac{\left|h_{2}\right|+\left|p_{2}\right|}{2\left|(\mu-2 \lambda)(1-2 \delta)^{k}\right|}, \sqrt{\frac{\left|h_{2}\right|^{2}+\left|p_{2}\right|^{2}}{2(\mu-2 \lambda)^{2}(1-2 \delta)^{2 k}}+\frac{(\mu-1)^{2}\left(\left|h_{1}\right|^{2}+\left|p_{1}\right|^{2}\right)^{2}}{16(\mu-\lambda)^{4}(1-2 \delta)^{2 k}}}\right] .
$$

This complete the proof.

\section{Corollaries and Consequences}

If we take $k=0$ in Theorem 2.1, then obtain following Corollary.

Corollary 3.1 [13] Let $f(z)$ of the form (1) be in the class $\Sigma_{M}^{h, p}(\mu, \lambda), \lambda \geq 1, \lambda>\mu, \geq 0$. Then

$$
\left|b_{0}\right| \leq \min \left[\sqrt{\frac{\left|h_{1}\right|^{2}+\left|p_{1}\right|^{2}}{2(\mu-\lambda)^{2}}}, \sqrt{\frac{\left|h_{2}\right|+\left|p_{2}\right|}{|(\mu-2 \lambda)(\mu-1)|}}\right]
$$


and

$$
\left|b_{1}\right| \leq \min \left[\frac{\left|h_{2}\right|+\left|p_{2}\right|}{2|(\mu-2 \lambda)|}, \sqrt{\frac{\left|h_{2}\right|^{2}+\left|p_{2}\right|^{2}}{2(\mu-2 \lambda)^{2}}+\frac{(\mu-1)^{2}\left(\left|h_{1}\right|^{2}+\left|p_{1}\right|^{2}\right)^{2}}{16(\mu-\lambda)^{4}}}\right] .
$$

Remark 3.1 Corollary 3.1 is an improvement result of result obtained by Orhan [10] in Theorem 1.2.

If we take $h(z)=p(z)=\left(\frac{1+\frac{1}{z}}{1-\frac{1}{z}}\right)^{\alpha}=1+\frac{2 \alpha}{z}+\frac{2 \alpha^{2}}{z^{2}}+\cdots(0<\alpha \leq 1, z \in \Delta)$, in Theorem 2.1, then we obtain following result.

Corollary 3.2 Let $f(z)$ of the form (1) belong to the class $\Sigma_{M}^{*}(k, \delta, \mu, \lambda, \alpha)$, then

and

$$
\left|b_{0}\right| \leq \min \left[\frac{2 \alpha}{\left|(\mu-\lambda)(1-\delta)^{k}\right|}, \frac{2 \alpha}{\sqrt{\left|(\mu-2 \lambda)(\mu-1)(1-\delta)^{2 k}\right|}}\right]
$$

$$
\left|b_{1}\right| \leq \min \left[\frac{2 \alpha^{2}}{\left|(\mu-2 \lambda)(1-2 \delta)^{k}\right|}, 2 \alpha^{2} \sqrt{\frac{1}{(\mu-2 \lambda)^{2}(1-2 \delta)^{2 k}}+\frac{(\mu-1)^{2}}{(\mu-\lambda)^{4}(1-2 \delta)^{2 k}}}\right] .
$$

Remark 3.2 Corollary 3.2 is an improvement result of result obtained by Bobalade and Sangle [4] in Theorem 2.6.

If we take $k=0$ in Corollary 3.2, then we obtain following Corollary.

Corollary 3.3 [13] Let $f(z)$ of the form (1) belong to the class $\Sigma_{M}^{*}(\mu, \lambda, \beta)$, then

and

$$
\left|b_{0}\right| \leq \min \left[\frac{2 \alpha}{(\lambda-\mu)}, \frac{2 \alpha}{\sqrt{|(\mu-2 \lambda)(\mu-1)|}}\right]
$$

$$
\left|b_{1}\right| \leq \min \left[\frac{2 \alpha^{2}}{(2 \lambda-\mu)}, 2 \alpha^{2} \sqrt{\frac{1}{(\mu-2 \lambda)^{2}}+\frac{(\mu-1)^{2}}{(\mu-\lambda)^{4}}}\right] .
$$

If we take $h(z)=p(z)=\frac{1+\frac{(1-2 \beta)}{z}}{1-\frac{1}{z}}=1+\frac{2(1-\beta)}{z}+\frac{2(1-\beta)}{z^{2}}+\cdots \quad(0 \leq \beta<1, z \in \Delta)$, in Theorem 2.1, then we obtain following result.

Corollary 3.4 Let $f(z)$ of the form (1) be in the class $\Sigma_{M}^{*}(k, \delta, \mu, \lambda, \beta)$, then

and

$$
\left|b_{0}\right| \leq \min \left[\frac{2(1-\beta)}{\left|(\mu-\lambda)(1-\delta)^{k}\right|}, \sqrt{\frac{4(1-\beta)}{\left|(\mu-2 \lambda)(\mu-1)(1-\delta)^{2 k}\right|}}\right]
$$

$$
\left|b_{1}\right| \leq \min \left[\frac{2(1-\beta)}{\left|(\mu-2 \lambda)(1-2 \delta)^{k}\right|}, 2(1-\beta) \sqrt{\frac{1}{(\mu-2 \lambda)^{2}(1-2 \delta)^{2 k}}+\frac{(1-\beta)^{2}(\mu-1)^{2}}{(\mu-\lambda)^{4}(1-2 \delta)^{2 k}}}\right] .
$$

Remark 3.3 Corollary 3.4 is an improvement result of result obtained by Bobalade and Sangle [4] in Theorem 2.3.

If we take $k=0$ in Corollary 3.4, then we get following Corollary .

Corollary 3.5 [13] Let $f(z)$ of the form (1) belong to the class $\Sigma_{M}^{*}(\mu, \lambda, \beta)$, then

and

$$
\left|b_{0}\right| \leq \min \left[\frac{2(1-\beta)}{(\lambda-\mu)}, 2 \sqrt{\frac{(1-\beta)}{|(\mu-2 \lambda)(\mu-1)|}}\right]
$$

$$
\left|b_{1}\right| \leq \min \left[\frac{2(1-\beta)}{(2 \lambda-\mu)}, 2(1-\beta) \sqrt{\frac{1}{(\mu-2 \lambda)^{2}}+\frac{(1-\beta)^{2}(\mu-1)^{2}}{(\mu-\lambda)^{4}}}\right] .
$$

\section{Acknowledgment:}

The authors wish to thank the referee, for the careful reading of the paper and for the helpful suggestions and comments. 


\section{References}

[1] Ai-Oboudi, F. M. (2004). On univalent functions defined by a generalized Salagean operator. Int.J. of Math. And Math. Sci., 27: 1429-1436.

[2] Brannan, D. A. and Taha, T. S. (1986). On some classes of bi-univalent functions. Studia Univ. Babes-Bolyai Math., 31(2): 70-77.

[3] Bobalade, D. D. and Sangle, N. D. (2019). Coefficient estimates for a certain subclass of bi-univalent functions defined by multiplier transformation. International Journal of Research and Analytical Reviews, 6(1): 123-128.

[4] Bobalade, D. D. and Sangle, N. D. (2021). Certain subclasses of bi-univalent and meromorphic functions associated with al-oboudi differential operator. International Journal of Mathematics Trends and Technology, 67(5): 86-89.

[5] Duren, P. L. (1983). Univalent functions (Grundlehren der Mathematischen Wissenschaften, Band 259, Springer-Verlag, New York, Berlin, Heidelberg and Tokyo).

[6] Frasin, B. A. and Aouf, M. K. (2011). New subclasses of bi-univalent functions. Appl. Math. Lett., 24: 1569-1573.

[7] Hamidi, S. G., Halim, S. A. and Jahangiri, J. M. (2013). Coefficient estimates for a class of meromorphic bi-univalent functions. C. R. Acad. Sci. Paris, Ser. I, 351: 349-352.

[8] Janani, T. and Murugusundaramoorthy, G. (2014). Coefficient estimates of meromorphic bi-starlike functions of complex order. Internat. J. Anal. Appl., 4(1): 68-77.

[9] Kubota, Y. (1977). Coefficients of meromorphic univalent functions. Kodai Mathematical Seminar Report, 28: 253-261.

[10] Orhan, H., Mangesh, N. and Balaji, V. K. (2014). Initial coefficient bounds for certain subclasses of meromorphic bi-univalent functions. Asian European J.Math., 7(1): 1-9.

[11] Patil, A.B. and Naik, U.H. (2017). Coefficient estimates for a new subclass of meromorphic bi-univalent functions defined by Al-Oboudi differential operator. Global Journal of Pure and Applied Mathematics, 13(9): 4405-4414.

[12] Safa S. and Ahmad Z. (2017). Coefficient estimates for certain subclass of meromorphic and bi-univalent functions. Commun. Korean Math. Soc., 32(2): 389-397.

[13] Saideh H. and Ahmad Z. (2019). Coefficients estimates for certain subclass of meromorphic and bi-univalent functions. Commun. Fac. Sci. Univ. Ank. Ser. Al Math. Stat., 68(1): 654-662.

[14] Schiffer, M. (1938). Surun probleme dextremum de la representation conforme. Bull. Soc. Math. France, 66: 48-55.

[15] Springer, G. (1951). The Coefficients problem for Schlicht mappings of the exterior of the unit circle. Trans. Amer. Math. Soc., 70: 421-450.

[16] Srivastava, H. M., Mishra, A. K. and Gochhayat, P. (2010). Certain subclasses of analytic and bi-univalent functions. Applied Mathematics Letters, 23: 1188-1192.

[17] Srivastava, H. M., Joshi, S.B., Joshi, S. S. and Haridas, P. (2016). Coefficient Estimates for Certain Subclasses of Meromorphically Bi-Univalent Functions. Palestine Journal of Mathematics, 5(1): 250-258.

[18] Suzeini, A.H., Samaneh, G. H. and Ravichandran, V. (2011). Coefficient Estimates for Meromorphic Bi-Univalent Functions. arXiv:1108.4089v1, 1-9. 